\title{
Plasma Treatment for Preparing Durable Water Repellent and Anti-Stain Synthetic Fabrics for Automotive Applications
}

\author{
Nicoletta De Vietro ${ }^{*}$, Luca Belforte ${ }^{2}$, Vito Lambertini' ${ }^{2}$, Bartolomeo Placenza², \\ Francesco Fracassi ${ }^{1}$ \\ ${ }^{1}$ Department of Chemistry, University of Bari Aldo Moro, Bari, Italy \\ ${ }^{2}$ Centro Ricerche FIAT (CRF) S.C.p.A., Torino, Italy \\ Email: ${ }^{*}$ nicoletta.devietro@uniba.it
}

Received 12 January 2015; accepted 26 May 2015; published 1 June 2015

Copyright (C) 2015 by authors and Scientific Research Publishing Inc.

This work is licensed under the Creative Commons Attribution International License (CC BY).

http://creativecommons.org/licenses/by/4.0/

c) (i) Open Access

\section{Abstract}

This paper describes the development of a plasma process to produce a durable water repellent and anti-stain thin film on synthetic textile, utilized for the upholstery in the automotive field. The coatings were deposited in non equilibrium low pressure plasmas fed with $1 \mathrm{H}, 1 \mathrm{H}, 2 \mathrm{H}$-perfluo-ro1-decene employing, as substrates, polyethylene terephthalate and polyethylene terephthalate thermo-coupled to polyurethane foam. It was found that the XPS F/C ratio of the deposit was higher than 1.4 and that the treated textile was always very hydrophobic (WCA $>140^{\circ}$ ) and oil resistant (motor oil $\mathrm{CA}>\mathbf{1 1 0}^{\circ}$ ), even after wear.

\section{Keywords}

Synthetic Textile, Automotive, Durable Water Repellent and Anti-Stain Character, Low Pressure Plasma, Fluorinated Coating

\section{Introduction}

The finishing processes of textiles devoted to improving the quality of the fabric and impart specific properties, such as hydrophobicity, hydrophilicity, anti-bacterical, flame retardant, shrink resistance, etc. [1]-[6], are becoming always and more important.

Both chemical and physical methods are generally used to obtain specific properties. The conventional treatments, including the widespread dip-coating and padding with chemicals, require the utilization of large amount

\footnotetext{
${ }^{*}$ Corresponding author.

How to cite this paper: De Vietro, N., Belforte, L., Lambertini, V., Placenza, B. and Fracassi, F. (2015) Plasma Treatment for Preparing Durable Water Repellent and Anti-Stain Synthetic Fabrics for Automotive Applications. Journal of Surface Engineered Materials and Advanced Technology, 5, 103-109. http://dx.doi.org/10.4236/isemat.2015.53012
} 
of chemicals (generally in solution) with important environmental impact. These treatments may also affect the mechanical properties of the fabrics as, for instance, reducing the durability and the comfort to wear.

Alternative techniques have been investigated, during the last years, with the aim of reducing the utilization of chemicals. Among these, plasma treatment attracts particular interest especially for its main peculiarities: the treatments interest only the uppermost layers of the fabric surface without modifying the bulk properties [7] and it is environmental friendly, since the use of chemicals is negligible [8]. The utilization of low pressure plasma processes, in particular, has been widely investigated for the modification of surface properties of textile composed by synthetic polymers and natural materials [1] [2] [8]. The surface modifications and properties depend on the feeding gas and on the operating conditions (input power, pressure, electrode geometry, etc.); a proper selection of these parameters allows to obtain different processes with the same experimental apparatus, i.e. etching, grafting, cross-linking and deposition [2].

Despite these advantages, there are only few commercial applications of plasma treatments in the textile field, such as the employment of the process to increase the wettability of the fabrics up to $160 \mathrm{~cm}$ width developed in a plant build up from Niekmi Institute [9]. In addition to the difficult scale-up from lab to industrial scale, this could be due to the short lifetime of the plasma treatments that very often do not meet the demands of the textile industry in terms of resistance to washing, to light, to perspiration, etc. [2].

Among the different textile properties that can be improved with the plasma technology, the water repellency and the resistance to motor oil stains are very important for automotive applications. Several research groups investigated the hydrophobicity enhancement of polymers and fabrics using plasmas fed with fluorocompounds, e.g. tetrafluoromethane $\left(\mathrm{CF}_{4}\right)$ [10], sulphur exafluoride $\left(\mathrm{SF}_{6}\right)$ [11], exafluoroethane $\left(\mathrm{C}_{2} \mathrm{~F}_{6}\right)$ [12], exafluoropropene $\left(\mathrm{C}_{3} \mathrm{~F}_{6}\right)$ [13], etc. It was found that plasma fed with small molecules (e.g. $\left.\mathrm{CF}_{4}, \mathrm{C}_{2} \mathrm{~F}_{6}\right)$ did not result in treatments of good durability, probably for the formation of short polymer segments dangling on the treated surface [11]. For example, a coating with F/C ratio of 1.04 deposited on silk and cotton in plasma fed with $\mathrm{C}_{3} \mathrm{~F}_{6}$ shows a good hydrophobic character (water contact angle, WCA higher than $120^{\circ}$ ), but suffers a partial loss after waterwashing and alcohol-extraction.

In this paper, the plasma enhanced-chemical vapor deposition (PE-CVD) with a large fluorinated molecule, the $1 \mathrm{H}, 1 \mathrm{H}, 2 \mathrm{H}$-perfluoro-1-decene, is studied in order to impart to the synthetic textile, employed for vehicle interiors in the automotive industry, durable water repellency and anti-stain character respect to motor oil.

It was found that when a $100 \mathrm{~nm}$ thick layer with a surface XPS (X-ray photoelectron spectroscopy) F/C ratio higher than 1.4 is deposited, the treated textile is very resistant to water (WCA $150^{\circ}$ ) and oil (motor oil contact angle, CA $120^{\circ}$ ). These properties are preserved also after usury caused by a test which simulates the wear suffered by a car seat during its use for the average lifetime of the vehicle.

\section{Materials and Methods}

\subsection{Low Pressure Plasma Treatment Experimental Conditions}

Treatments were performed on as received substrates $(80 \times 80 \mathrm{~mm})$ made of polyethylene terephthalate (PET) and of polyethylene terephthalate thermo-coupled to $5 \mathrm{~mm}$ tick polyurethane (PU) foam. These materials are commonly used by the automotive industry for the production of car and commercial vehicles interiors.

The experiments were carried out in the stainless steel, parallel plate, low pressure reactor depicted in Figure 1 .

It consists of a cylindrical stainless steel chamber (internal diameter $=200 \mathrm{~mm}$; height: $400 \mathrm{~mm}$ ) equipped with two stainless steel circular electrodes (diameter $=150 \mathrm{~mm}$, inter-electrode distance $=40 \mathrm{~mm}$ ), pumped by a turbomolecular-rotary pumping system. The pressure was measured and controlled with a baratron gauge (MKS) and a manual throttle valve, respectively. The upper electrode was connected to a $13.56 \mathrm{MHz}$ radio frequency (RF) power supply though an automatic L-type matching network unit, while, the lower electrode, on which the textile samples were positioned during the deposition processes, was grounded.

The experiments were performed at 2.0 Pa and $10-100 \mathrm{~W}$ of input power, with the flow rate of $1 \mathrm{H}, 1 \mathrm{H}, 2 \mathrm{H}-$ perfluoro-1-decene vapour (Sigma Aldrich, purity > 99.9\%) fixed, through a needle valve, at $15 \mathrm{sccm}$ (standard cubic centimeters for minute).

\subsection{Surface Characterization of Plasma Modified Textiles}

The thickness of the coating was determined by means of a KLA Tencor D120 profilometer on Si-c (100) sub- 


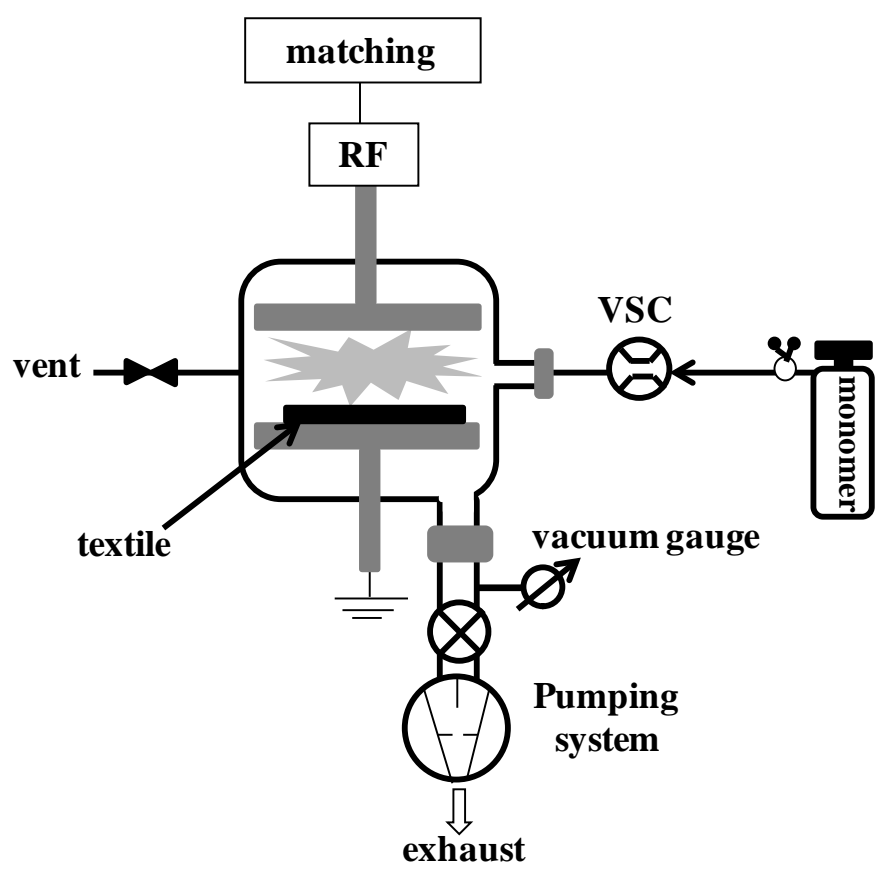

Figure 1. Schematic of the low pressure plasma reactor.

strates (flat reference material), partially masked during the deposition process.

The chemical characterization of treated and untreated samples was performed by means of X-ray Photoelectron Spectroscopy, using a Theta Probe spectrometer (Thermo Electron Corporation) equipped with monochromatic $\mathrm{Al} \mathrm{K} \alpha \mathrm{X}$-ray source $(1486.6 \mathrm{eV})$, operated at a spot size of $300 \mu \mathrm{m}$ corresponding to a power of $70 \mathrm{~W}$. Survey $(0-1200 \mathrm{eV})$ and high resolution spectra were recorded in FAT (fixed analyzer transmission) mode at a pass energy of 200 and $100 \mathrm{eV}$, respectively. All spectra were acquired at a takeoff angle of $37^{\circ}$. A flood gun was used to balance surface charging. The C1s signal for the hydrocarbon component $(285.0 \mathrm{eV})$ was used as internal standard for charging correction. Atomic percentages were calculated from the high resolution spectra using the Scofield sensitivity factors set in the XPS data processing software and a non-linear Shirley background subtraction algorithm. Repeated measurements allowed registering a maximum relative standard deviation of about $3 \%$.

Surface morphology of treated and untreated textile was evaluated using a Zeiss SUPRA ${ }^{\mathrm{TM}} 40$ field emission scanning electron microscope (FESEM). Images were acquired after chrome metallization at a tilt angle of $0^{\circ}$ at an acceleration voltage of $2 \mathrm{KV}$.

The water and oil wettability of textile samples was studied by static and/or dynamic water and motor oil (Red Line synthetic oil, 5w40) contact angle measurements, performed by means of an automatic goniometer (Nordtest), utilizing droplets of $2 \mu \mathrm{l}$ of volume. The contact angle values were obtained averaging three measurements conducted in different parts of the same sample and on three different samples.

The wear resistance of treated and untreated surfaces was evaluated with a "Cesconi" abrasion tester, according to UNITEX 7858 rule, which reproduces the mechanical stress suffered by a car seat during its normal use for the average lifetime of the vehicle.

The test was carried out employing an abrasimeter general utilized for texting textile, leather, imitation leather (PU; polyvinyl chloride, PVC), non-woven, ceramic, rubber, etc. The abrading unit was made of PET textile fixed on a rotating plate. The substrate to test was located on a rotating "satellite" situated under the rotating plate, as schematized in Figure 2.

The "satellite" and the rotating plate rotated with different speeds, in order to ensure a uniform ablation of the analyzed sample.

A wear cycle of 3000 revolutions was performed with a load of $1 \mathrm{Kg}$, according to the internal FIAT validation test. Three different samples for each condition were examined. 


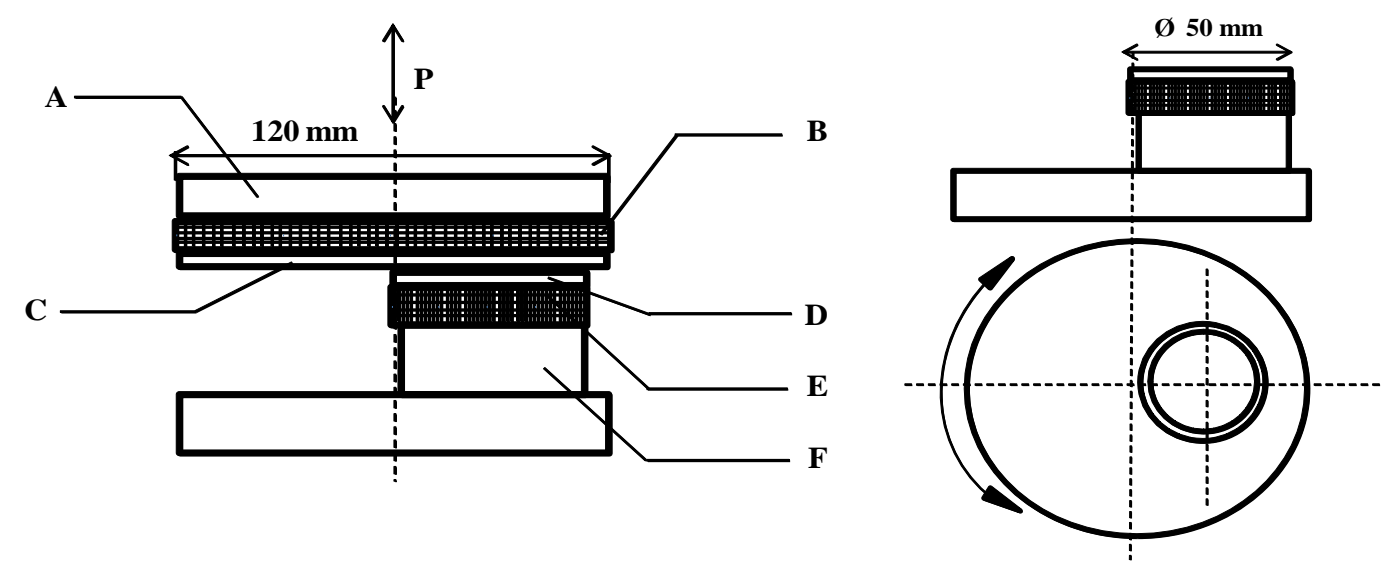

Figure 2. Scheme of the wear resistance test device. The substrate to test (D) is mounted on the satellite (F) through a substrate holder $(\mathrm{E})$, while the abrading fabric $(\mathrm{C})$ is fixed on the rotating plate $(\mathrm{A})$ employing the substrate holder (B), on which the weight $(\mathrm{P})$ is applied.

\section{Results and Discussion}

The XPS atomic composition and the F/C ratio of the plasma treated uncoupled PET are reported in Table 1, as a function of the input power. The chemical composition of virgin PET is also quoted for comparison.

Under the deposition conditions utilized, the effect of input power on the surface composition of the fluoropolymer coatings is very low. The F/C ratio varies in a narrow range (i.e. 1.47 - 1.65), the highest value is obtained at the lowest input power $(20 \mathrm{~W})$, probably for the light plasma fragmentation which preserves the chemical integrity of the monomer. The low effect of input power on the chemical composition of the coatings is reflected also on their hydrophobic/oleophobic behavior. As it can be appreciated in Figure 3, in fact, all samples have similar oil and water contact angles, with a slight reduction by increasing the input power (i.e. decrease of the fluorine content).

Untreated textile, on the other hand, was super-hydrophilic and super-oleophilic, in fact, contact angle measurements were not possible, because the drops of water and oil were instantaneously adsorbed by the sample.

SEM observations showed that all plasma coatings were compact and uniformly covered the fabric.

In order to evaluate the utilization of the optimized plasma treatment to produce water repellent and anti-stain fabric for seat upholstery in automotive field, the experiments were repeated under the same conditions on thermo-coupled PET textile. No difference, respect to the uncoupled substrate, was detected for the water and motor oil contact angle values. This is an important result because it means that the plasma treatment can be performed after the thermo-coupling process between the PET fabric and the polyurethane foam. Since the plasma treatment in part interests also the back side of the fabric, whether the treatment was carried out before the thermo-coupling step, it would result in pour textile-foam adhesion.

The wear resistance of the plasma coated thermo-coupled fabrics was evaluated with the "Cesconi" test described in the section "Materials and Methods". Figure 4 reports the static contact angles of water and oil, measured after the wear test. All values were collected after 120 seconds of contact of the water/oil drop on the textile surface.

All the samples preserved the hydrophobic character after the test even though, by the comparison with Figure 3, it appears that, except for the sample treated at $100 \mathrm{~W}$, the WCA values decrease after the wear test. Also the static motor oil CA values decrease after the wear test; the lowest reduction is shown by the sample treated at highest input power value $(100 \mathrm{~W})$.

Figure 5 clearly shows that the samples with the coatings deposited at 50 and $100 \mathrm{~W}$ exhibit a good and stable oleophobic character, also after the wear test. On the contrary, the fabrics coated at 20 and $30 \mathrm{~W}$, despite their initial super-hydrophobic/oleophobic behavior, after the wear test became oleophylic.

The loss of the hydrophobic and oleophobic character suffered by the plasma modified fabrics can be due to mechanical damage caused by the "Cesconi" test. This damage is lower for the coatings deposited at 50 and 100 $\mathrm{W}$, probably for the higher cross-linking promoted by the high input power which results in higher precursor 
fragmentation and stronger ion bombardment. This is confirmed by the SEM images acquired after the wear test (Figure 6), that clearly show as the coating deposited at $100 \mathrm{~W}$ does not suffer serious mechanical damages, while the coating deposited at $20 \mathrm{~W}$ is almost completely destroyed after wear test. Also XPS analyses are in agreement with this conclusion, in fact, while the surface chemical composition of the coating deposited at 100 W shows small changes after the wear test (e.g. the fluorine content decreases from 58\% to 51\%), the XPS surface

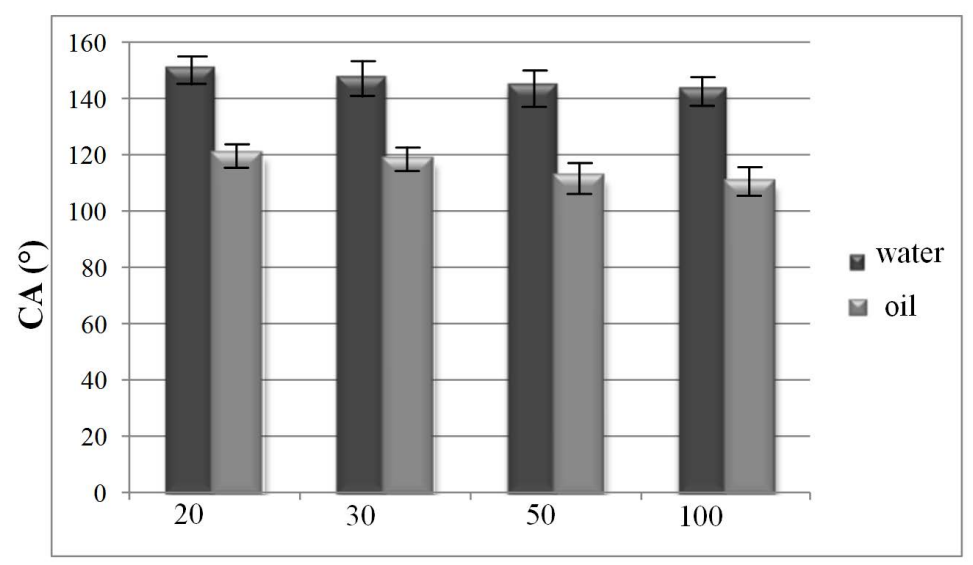

input power (W)

Figure 3. Water and oil contact angle values for plasma coated uncoupled PET textile vs. input power.

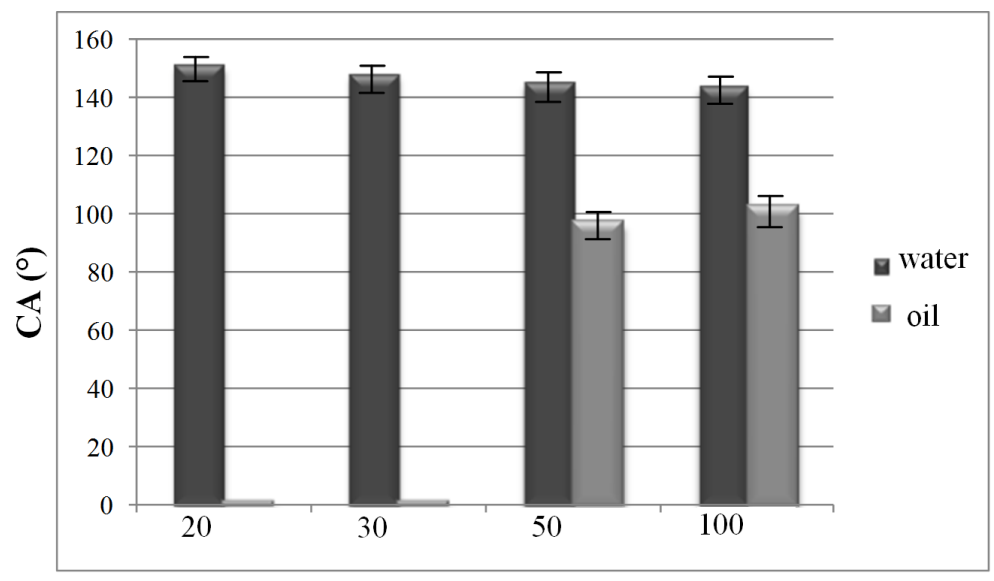

input power (W)

Figure 4. WCA and motor oil contact angle values measured after 120 seconds of contact of the worn thermo-coupled textile with the drop. The oil contact angle for the samples treated at $20 \mathrm{~W}$ and $30 \mathrm{~W}$ was zero.

Table 1. XPS results of uncoupled PET textile, as a function of input power (coating thickness, $100 \mathrm{~nm}$ ).

\begin{tabular}{ccccc}
\hline Input Power & $\mathbf{C} \%$ & $\mathbf{O} \%$ & $\mathbf{F} \%$ & F/C \\
\hline Virgin Textile & $74 \pm 1$ & $26 \pm 2$ & $/$ & $/$ \\
$20 \mathrm{~W}$ & $37 \pm 3$ & $2 \pm 1$ & $61 \pm 3$ & 1.65 \\
$30 \mathrm{~W}$ & $38.0 \pm 0.7$ & $3.1 \pm 0.5$ & $58.9 \pm 0.6$ & 1.55 \\
$50 \mathrm{~W}$ & $39 \pm 2$ & $2.1 \pm 0.4$ & $58.9 \pm 0.5$ & 1.51 \\
$100 \mathrm{~W}$ & $39.5 \pm 0.5$ & $2.5 \pm 0.5$ & $58 \pm 2$ & 1.47 \\
\hline
\end{tabular}


chemical composition of the coating deposited at $20 \mathrm{~W}$ varies considerably after the wear test (e.g. the fluorine content decreases from $61 \%$ to $13.7 \%)$.

These experimental evidences allow to conclude that PET textile, thermo coupled with PU foam and coated with $100 \mathrm{~nm}$ of a fluorinated thin film deposited in low pressure plasma at $100 \mathrm{~W}$, can be considered as possible, durable, water repellent and anti-stain material for automotive uses.

\section{Conclusions}

In this paper, a durable water/motor oil resistant fluorinated coating $(\mathrm{F} / \mathrm{C} \geq 1.47)$ was deposited in low pressure plasma conditions. The optimized film was used to cover the thermo coupled with $5 \mathrm{~mm}$ of polyurethane foam synthetic textile, generally used in automotive field. The obtained fabrics were wear resistant, particularly if they

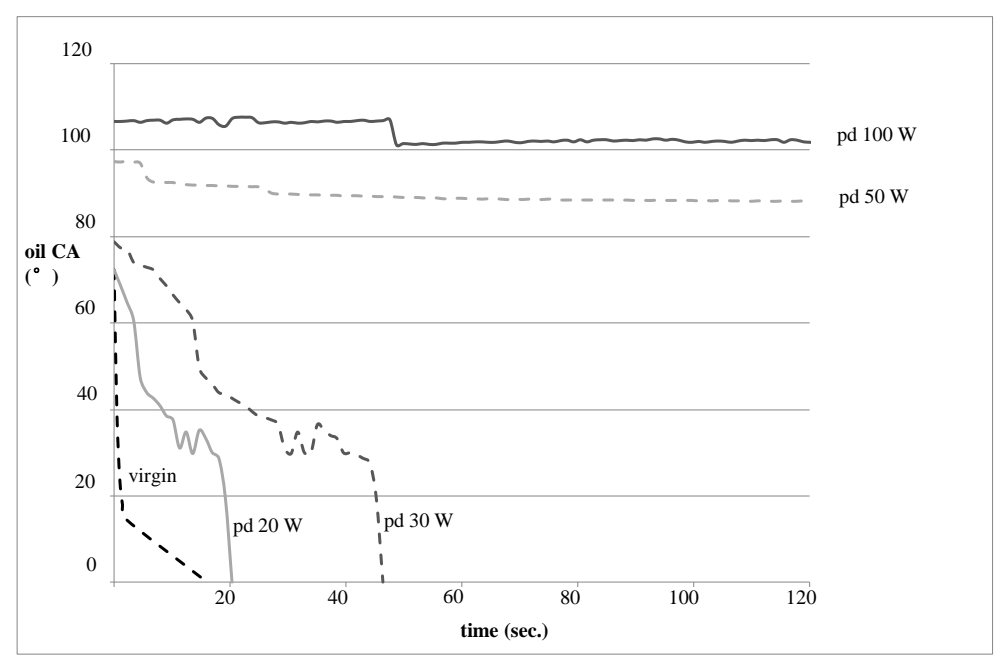

Figure 5. Dynamic motor oil contact angle values after "Cesconi” test for plasma coated thermo-coupled textile.
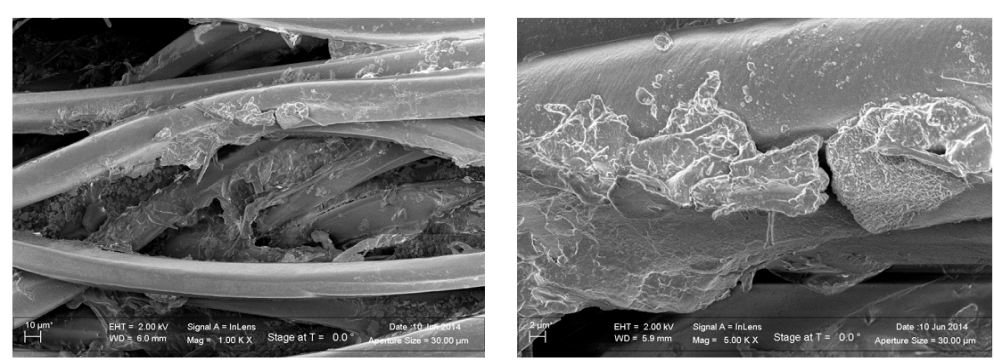

(a)
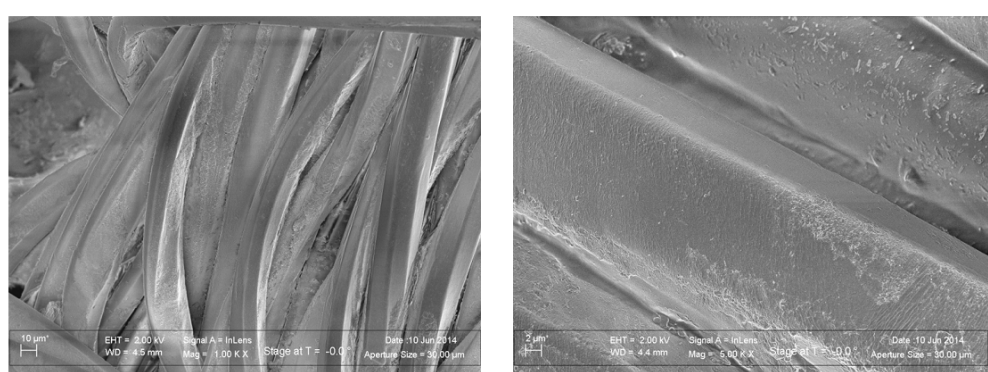

(b)

Figure 6. SEM images (magnification 1 and $5 \mathrm{Kx}$ ) of PET textile, thermo coupled with $5 \mathrm{~mm}$ of PU foam, coated with plasma coatings after "Cesconi" test. Plasma coating obtained at $20 \mathrm{~W}$ (a) and $100 \mathrm{~W}$ (b) of input power value. 
were modified at high value of input power.

The optimized deposition process can be potentially employed in automotive field to obtain durable, water repellent and anti-stain textiles useful for the realization of car and commercial vehicle interiors.

\section{Acknowledgements}

This research was financially supported by MIUR: PON01_02239 "Materiali avanzati per trasporti ecosostenibili" (MATRECO), PONa3_00369 "Laboratorio SISTEMA" and by Regione Puglia within the "Accordo di programma quadro ricerca scientifica, II atto integrativo", project n. 51 "Laboratorio pubblico di ricerca industriale pugliese dei plasmi” (LIPP).

Dr. Piera Bosso is gratefully acknowledged for her technical support and Dr. Fabio Palumbo for the SEM images.

\section{References}

[1] Chan, C.-M. (1994) Polymer Surface Modification and Characterization. Hanser Publishers, Munich.

[2] Vohrer, U., Muller, M. and Oehr, C. (1998) Glow-Discharge Treatment for the Modification of Textiles. Surface and Coatings Technology, 98, 1128-1131.

[3] Hesse, A., Thomas, H., Hocker, H. and Zero, A.O.X. (1995) Shrink Proofing Treatment for Wool Top and Fabric: Part I: Glow Discharge Treatment. Textile Research Journal, 65, 355-361.

[4] Zhang, W., Luo, Y., Wang, H., Jiang, J., Pu, S. and Chu, P.K. (2008) Ag and Ag/ $\mathrm{N}_{2}$ Plasma Modification of Polyethylene for the Enhancement of Antibacterial Properties and Cell Growth/Proliferation. Acta Biomaterialia, 4, 2028-2036.

[5] Tsafack, M.J. and Levallois-Grutzmacher, J. (2006) Flame Retardancy of Cotton Textiles by Plasma-Induced GraftPolymerization (PIGP). Surface and Coatings Technology, 201, 2599-2610.

[6] Hossain, M.M., Herrmann, A.S. and Hegemann, D. (2006) Plasma Hydrophilization Effect on Different Textile Structures. Textile Research Journal, 65, 299-307.

[7] Poll, H.U., Schladitz, U. and Schreiter, S. (2001) Penetration of Plasma Effects into Textile Structures. Surface and Coatings Technology, 142-144, 489-493.

[8] Yip, J., Chan, K., Sin, K.M. and Lau, K.S. (2002) Low Temperature Plasma-Treated Nylon Fabrics. Journal of Materials Processing Technology, 123, 5-12.

[9] N.N. ITB Veredlung (1995) 22-24.

[10] Sigurdsson, S. and Shishoo, R. (1997) Surface Properties of Polymers Treated with Tetrafluoromethane Plasma. Journal of Applied Polymer Science, 66, 1591-1601.

[11] Hodak, S.K., Supasai, T., Paosawatyanyong, B., Kamlangkla, K. and Pavarajarn, V. (2008) Enhancement of the Hydrophobicity of Silk Fabrics by $\mathrm{SF}_{6}$ Plasma. Applied Surface Science, 254, 4744-4749.

[12] Sun, D. and Stylios, G.K. (2006) Fabric Surface Properties Affected by Low Temperature Plasma Treatment. Journal of Materials Processing Technology, 173, 172-177.

[13] Li, S. and Jinj, D. (2007) Improvement of Hydrophobic Properties of Silk and Cotton by Hexafluoropropene Plasma Treatment. Applied Surface Science, 253, 5051-5055. 